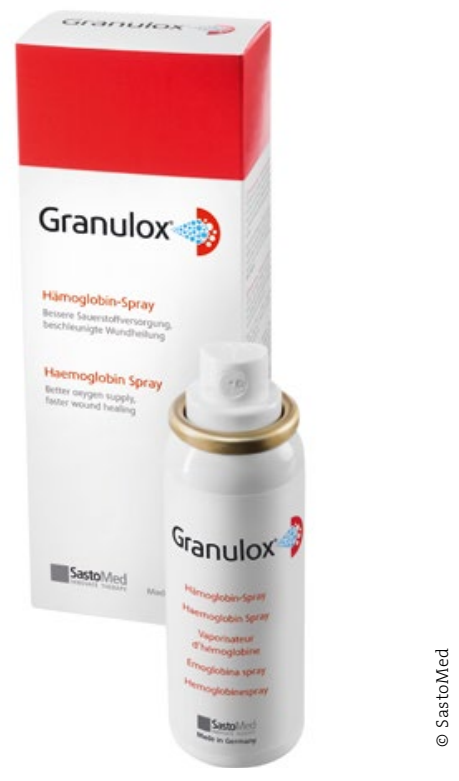

\title{
Leitlinie zur systemischen und lokalen Sauerstoffbehandlung
}

") Rund vier Millionen Patienten leiden in Deutschland an schlecht heilenden und chronischen Wunden - entsprechend wichtig ist eine hochwirksame und kosteneffiziente Therapie. Da einer stagnierenden Wundheilung meist eine Hypoxie, also ein Mangel an Sauerstoff, zugrunde liegt, wird weltweit fieberhaft an der Entwicklung neuer Sauerstofftherapien gearbeitet. Auf der diesjährigen European Wound Management (EWMA) Konferenz in Amsterdam wurde Anfang Mai das Dokument „Use of oxygen therapies in wound healing " veröffentlicht und in einer Key Session von Experten vorgestellt. Neben der hyperbaren Sauerstofftherapie (HBOT) werden darin auch topische Sauerstofftherapien (TOT) valide und umfassend untersucht.

Die Veröffentlichung des neuen EWMA-Dokuments ist ein Durchbruch für die Sauerstofftherapie chronischer Wunden. Erstmals werden darin alle aktuell gängigen HBOT- und TOT-Behandlungsformen aufgeführt, analysiert und auf Basis der vorliegenden wissenschaftlichen Evidenz bewertet. Rund drei Jahre lang arbeitete ein Spitzenteam aus internationalen Experten gemeinsam mit den Herausgebern Prof. Dr. Finn Gottrup und Prof. Dr. Joachim Dissemond an der umfassenden Untersuchung. Gemeinsam mit ihren internationalen Kollegen Carol Baines, Sharon Hunt, Peter $\emptyset$ strup Jensen und Jacek Kot präsentierten die beiden Herausgeber auf der EWMA ihre theoretimit Sauerstofftherapien und sprachen Behandlungsempfehlungen aus.

Laut der Expertengruppe zählt Granulox ${ }^{\circledR}$ zu den besten verfügbaren Therapien für die Sauerstoffversorgung von chronischen Wunden und erhält daher die Bewertung GRADE 1B. Mit dem bewährten GRADE-Ansatz (Grades of Recommendation Assessment, Development and Evaluation) bewerten die Experten die Qualität der existierenden Studien- und Forschungsergebnisse und sprechen dementsprechend starke oder schwache Empfehlungen für die Produkte aus. Aktuell ist die GRADE 1B-Bewertung die höchste Auszeichnung für ein TOTProdukt und wird neben Granulox ${ }^{\circledR}$ nur einem weiteren Therapiesystem zuteil. Neben der hohen Benutzerfreundlichkeit zeichnet sich das Hämoglobinspray durch niedrige Be- handlungskosten aus und kann von Patienten schnell und einfach selbst angewendet werden. „Für uns ist das EWMA-Dokument die Bestätigung unserer langen und kontinuierlichen Arbeit, die bahnbrechenden Behandlungserfolge von Granulox ${ }^{\circledR}$ wissenschaftlich sauber nachzuweisen. Es verbindet eine Vielzahl von klaren Vorteilen - und das für gleich mehrere Interessensgruppen. So profitieren Patienten und Anwender dank der verbesserten Sauerstoffzufuhr von einer beschleunigten Wundheilung. Kostenträgern wiederum bietet es sinkende Behandlungskosten, wie das EWMADokument ebenfalls deutlich zeigt", so Michael Sander, Geschäftsführer der SastoMed GmbH.

\section{www.sastomed.de}

\section{Sauberkeit und Hygiene}

\section{Mit Dampfreiniger im Pflegeheim}

» Dass Sauberkeit und Top-Hygiene im Seniorenheim nicht auf Kosten der

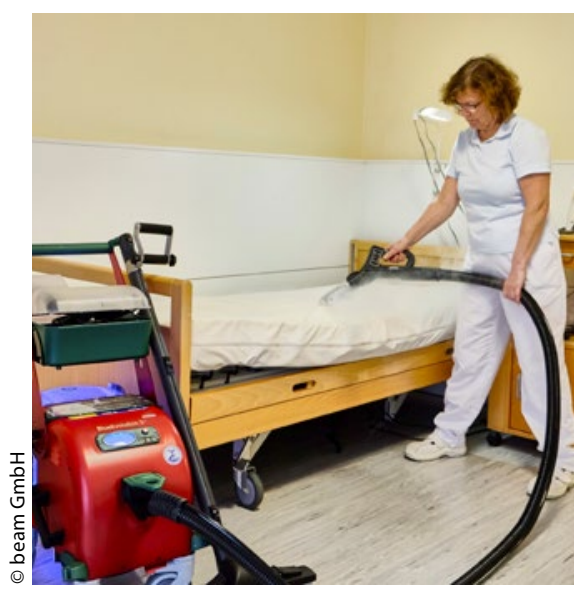

Betreuung der Bewohner gehen müssen, beweist das Ludwig-Steil-Haus des Evangelischen Johanneswerks in Herne: Seit die Mitarbeiter bei der Reinigung von Zimmern, Gemeinschaftsräumen, Sanitäranlagen und in der Küche die natürliche Reinigungskraft des innovativen Dampfsaugsystems Blue Evolution S+ der beam GmbH einsetzen, haben sie wieder mehr Zeit für ihre Patienten. Der effiziente, HACCPzertifizierte Saubermacher dampft und saugt in einem Arbeitsgang und spart dem Reinigungsteam damit bis zu $60 \%$ der Zeit, die bislang fürs Saubermachen nötig war. So haben die Mitarbeiter wieder mehr Zeit für die Bewohner und gleichzeitig auch die
Hygiene im Haus fest im Griff. Weiteres Plus: Die Geräte der Blue-EvolutionSerie arbeiten ausschließlich mit heißem Wasserdampf und verzichten komplett auf chemische Reinigungsmittel.

Als Besonderheit wartete der Blue Evolution zudem mit einem BlaulichtEffekt auf, bei dem Keime keine Chance haben. Denn bei jedem Saugvorgang landen die gelösten Schmutzpartikel im Wasserfilter und werden dort im Wasser gebunden. Die im Schmutzwasser enthaltenen Keime werden dann über das Blaulicht abgetötet und die Luft so effizient mit gereinigt.

www.beam.de 